\title{
De la epidemiología psiquiátrica a la psicología positiva. Historia de la trayectoria científica de María Martina Casullo
}

From a Psychiatric Epidemiology to a Positive Psychology. History of the Scientific Trajectory of María Martina Casullo

Recibido: enero 14 de 2014 | Revisado: junio 8 de 2014 | Aceptado: julio 17 de 2014

LUCIANA MARIÑELARENA-DONDENA **

Universidad Nacional de San Luis, Argentina

doi:10.11144/Javeriana.upsy13-5.eppp

Para citar este artículo: Mariñelarena-Dondena, L. (2014). De la epidemiología psiquiátrica a la psicología positiva. Historia de la trayectoria científica de María Martina Casullo. Universitas Psychologica, 13(5), 1893-1904. http://dx.doi.org/10.11144/ Javeriana.upsy13-5.eppp

Becaria Interna de Postgrado Tipo II del Consejo Nacional de Investigaciones Científicas y Técnicas (CONICET). Facultad de Psicología. Dirección Postal: Belgrano 260, San Luis, Argentina. Código Postal: D5700HHW. Correo electrónico: lucianamd.psico@gmail.com

\section{RESUMEN}

La presente investigación de carácter historiográfico examina un momento particular de la trayectoria científica de María Martina Casullo (19402008). En primer lugar, se revisan sus estudios sobre los factores de riesgo en adolescentes, realizados a partir del año 1985 desde una perspectiva epidemiológica-preventiva. En segundo lugar, se analiza cómo dichos estudios epidemiológicos sobre depresión, riesgo suicida y malestar psicológico en adolescentes fueron el puntapié inicial para el análisis de los procesos salugénicos o protectores que impedían que las personas enfermaran aún frente a situaciones ambientales adversas. Finalmente, se concluye que estas investigaciones constituyen el principal antecedente del campo, que posteriormente Casullo definiera como psicología salugénica o positiva, en la Argentina a finales del siglo XX. De esa manera, se cuestiona críticamente la tendencia naturalizada y whig que no se ha interesado en hallar las condiciones ideológicas y políticas de la emergencia de la psicología positiva. Palabras clave

epidemiología psiquiátrica; psicología positiva; enfoque salugénico; historia de la psicología; Argentina

\section{A B S T R A C T}

The purpose of this historiographic paper is to carry out a critical analysis about one particular moment of the scientific trajectory of María Martina Casullo (1940-2008). In the first place, the article reviews her studies about the risk factors in adolescents from an epidemiologic-preventive approach since 1985. Secondly, the work points out how these epidemiologic studies concerning depression, suicidal risk and psychological unease were the kickoff for the analysis of the salutogenic or protective processes that prevent people to fall ill even when facing adverse environmental situations. Finally, this study concludes that these researches are the main record of the field that subsequently Casullo defined as salutogenic or positive psychology in Argentine at the end of 20th century. Thus, this paper critically analyzes the naturalized and whig tradition in psychology that has neglected the ideological and political conditions of the emergence of positive psychology. Keywords

psychiatric epidemiology; positive psychology; salutogenic approach; history of psychology; Argentine 
En la actualidad, las investigaciones históricas realizadas desde una perspectiva crítica, generalmente denominada "nueva historia", centran su interés en la influencia que ejercen los factores sociales y el contexto cultural en el desarrollo de la disciplina psicológica (Danziger, 1979; Harris, 1999). En este marco, pierden relevancia las iniciativas individuales y, en consecuencia, las biografías son relegadas a un segundo plano.

No obstante Laura Ball (2012), joven investigadora de la Universidad de York que participa de las corrientes críticas de la historiografía de la psicología, sostiene que es posible estudiar la historia de las figuras eminentes desde una perspectiva social e históricamente informada, evitando caer así en el culto de los grandes héroes. En este contexto, la presente investigación historiográfica examina un momento particular de la trayectoria científica de María Martina Casullo (1940-2008), quien fuera hasta su prematuro fallecimiento Investigadora Principal del Consejo Nacional de Investigaciones Científicas y Técnicas (CONICET), Profesora Consulta Titular de la Facultad de Psicología de la Universidad de Buenos Aires y Directora del Doctorado en Psicología de la Universidad de Palermo. Concretamente, se analizan sus estudios sobre los factores de riesgo en adolescentes, realizados desde una perspectiva epidemiológica-preventiva, a partir del año 1985. Los mismos pueden ser considerados como el principal antecedente de los posteriores desarrollos en el campo de la psicología salugénica o positiva en la Argentina a finales del siglo XX.

Nos interesa centrarnos en la personalidad de María Martina Casullo, porque constituye un testimonio clave que permite cuestionar las historias whig y naturalizadas acerca de la emergencia de la psicología positiva. En los últimos años, varios estudios han analizado los componentes ideológicos, los condicionamientos sociales y políticos que facilitaron la emergencia de esta corriente en Estados Unidos de Norteamérica (Christopher, Richardson, \& Slife, 2008; Gancedo, 2008; Held, 2004).

En ese contexto general, dentro del desarrollo de la historia de la psicología en América Latina -desarrollo desigual y conflictivo-, se pueden señalar otros cuestionamientos a la narración histórica legitimante de la psicología positiva. Precisamente, consideramos que la trayectoria de María Martina Casullo, personalidad central del campo de la evaluación psicológica en Argentina y figura destacada en el proceso de constitución de las carreras de psicología luego de la dictadura militar (Casullo, 1996), constituye un testimonio clave para analizar la historia de la psicología positiva que, al menos en la recepción argentina del movimiento, evidencia continuidades y rupturas en el contexto de todo un programa previo de investigación y de intervención en el campo de la psicología y de la salud mental (Casullo, 1992, 1998; Conti, 2010).

El momento temporal objeto de análisis se ubica dentro del período que Hugo Klappenbach (2006a) denomina de la plena institucionalización de la psicología, propuesto en su periodización de la psicología en Argentina, considerando que este se iniciaría aproximadamente hacia 1983 y se extendería hasta nuestros días. Cabe destacar dos características de tal período: la primera, la familiarización de la psicología local con instituciones internacionales como la American Psychological Association y la segunda, el mayor pluralismo teórico y profesional, por ejemplo, los desarrollos en psicología sistémica y cognitiva, aun cuando no desaparezca enteramente el marcado perfil clínico de sesgo psicoanalítico en la enseñanza de la psicología en nuestro país.

\section{Una breve introducción al concepto de psicología positiva}

En los albores del siglo XXI, Casullo planteó la existencia de "un nuevo paradigma de reflexión teórica y metodológica" (Casullo, 2000, p. 341) al que denominó psicología salugénica o positiva, cuyo propósito consistía, como su nombre lo indica, en estudiar las dimensiones positivas o salugénicas de la personalidad. En este marco, se destacaban sus investigaciones sobre los factores protectores o generadores de salud en la adolescencia (MariñelarenaDondena, 2012a).

Es interesante constatar un cierto clima de ideas que promovían los enfoques centrados en la salud. En efecto, paralelamente a los estudios de Casullo, en Estados Unidos de Norteamérica surgía la psi- 
De la epidemiología psiquiátrica a la psicología positiva. Historia DE la trayectoria Científica de María Martina Casullo

cología positiva. La tradición institucional dentro de la misma, señala que dicho movimiento tuvo su origen en el año 1998, en el discurso inaugural de Martin Seligman como presidente de la American Psychological Association (APA). La nueva corriente psicológica centraba su interés en cuatro pilares de estudio: las emociones positivas, los rasgos individuales positivos, las instituciones que promovían los dos primeros y los vínculos positivos (Castro Solano, 2010; Seligman \& Csikszentmihalyi, 2000).

En el año 2011, Seligman presentaba su teoría del bienestar, definiendo este último como un constructo multidimensional compuesto por cinco elementos: las emociones positivas, el compromiso, los vínculos positivos, el significado y el logro. El objetivo final de la psicología positiva consistía en incrementar estos cinco elementos integrantes del bienestar, con el fin de promover el florecimiento humano (human flourishing).

Por otro lado, es necesario recordar que en los años 80 del siglo XX surgió el concepto de promoción de la salud. La Carta de Ottawa define las prácticas promotoras de salud como:

El proceso de capacitar a las personas para aumentar el control sobre su salud y mejorarla. Para llegar a un estado de completo bienestar físico, mental y social, un individuo o grupo debe poder identificar y realizar sus aspiraciones, satisfacer sus necesidades, y manejar o transformar su ambiente. (Organización Mundial de la Salud [OMS], 1986, p. 1)

En ese marco, Bang (2014) remarca la importancia de la promoción de salud mental comunitaria como una estrategia que permite articular la atención primaria de salud y la salud mental. Por su parte, la OMS sostiene que las actividades de promoción de la salud mental deben tener como objetivo lograr el óptimo desarrollo psicológico y psicofisiológico de los individuos (OMS, 2004a, 2004b).

Por la misma época, emergió el enfoque salugénico dentro del cual se inscribía la psicología positiva. En el desarrollo del mismo se han destacado tres grandes hitos: la psicología humanística, las denominadas investigaciones independientes sobre aspectos funcionales del psiquismo y la orientación salugénica propuesta por Aaron Antonovsky. Asimismo, diferentes autores sostenían que la psicología humanística constituía uno de los principales antecedentes de esta nueva corriente psicológica (Gancedo, 2008; Linley, Joseph, Harrington, \& Wood, 2006; Mariñelarena-Dondena \& Gancedo, 2011).

Por otro lado, se han remarcado algunos de los problemas epistemológicos posiblemente no resueltos en las producciones de los autores más destacados del movimiento (Mariñelarena-Dondena, 2012b). En primer término, se ha puesto de manifiesto la impronta del contexto socio-históricocultural en el cual surge, destacándose como dos de sus rasgos centrales el positivismo y el pragmatismo norteamericanos (Gancedo, 2008; Held, 2004). También se ha señalado que apoyaba y promovía determinados valores morales representativos de la cultura norteamericana, fuertemente influenciados por el individualismo (Christopher et al., 2008). En segundo término, se ha afirmado que el campo de la psicología positiva carecía de una teoría unificada y coherente que guiara las investigaciones (Gancedo, 2008). En esa dirección, Linley et al. (2006) hacían hincapié en que la psicología positiva debía prosperar hacia una psicología unificada e integrada, definiéndola como el estudio científico del funcionamiento humano óptimo. Por último, diferentes autores sostenían que el éxito de la psicología positiva consistía paradójicamente en su desaparición como movimiento, quedando integrada en la corriente principal de la psicología (Diener, 2003; King, 2003; Sternberg \& Grigorenko, 2001; Vázquez, 2006).

Investigaciones anteriores afirman que el movimiento de la psicología positiva fue tempranamente recepcionado en la Argentina precisamente debido a los intereses intelectuales e institucionales de María Martina Casullo en el campo del enfoque salugénico (Gancedo, 2008; Mariñelarena-Dondena, 2012a). En este contexto, aun sin caer en esos mitos fundacionales característicos de la historiografía whig, puede apreciarse que el trabajo de Casullo Psicología salugénica o positiva. Algunas reflexiones (Casullo, 2000) constituye un punto de inflexión en la recepción y posterior desarrollo de la psicología positiva en la Argentina. 


\section{Investigaciones desde una perspectiva epidemiológica-preventiva}

Tradicionalmente, se ha definido a la epidemiología como 'el estudio de la distribución de una enfermedad en una comunidad en un momento dado'; asimismo, busca conocer las condiciones y los factores que han determinado dicha distribución de la enfermedad (Greenberg \& Getting, 1957; Lilienfeld, 1957). En esa dirección y dado que la psiquiatría concibe al hombre como una entidad bio-psico-social, la epidemiología psiquiátrica constituye 'la disciplina que investiga la relación existente entre los desórdenes mentales y los factores socio-culturales' (Leighton, Clausen, \& Wilson, 1957; Leighton \& Huges, 1961).

En relación a la prevención de la enfermedad, existe consenso en que la misma abarca tanto las medidas destinadas a evitar su aparición, eliminando o reduciendo los factores de riesgo, como también las acciones que buscan proteger a los grupos más vulnerables y mitigar las consecuencias de las patologías que ya se han desencadenado.

En ese marco, tanto Caplan (1973) como Leavell y Clark (1965) propusieron categorías similares para clasificar la prevención de la enfermedad de acuerdo al momento en el cual se lleven a cabo las intervenciones. Estos autores diferencian tres tipos de prevención:

1. Primaria: busca suprimir los factores de riesgo y brindar las medidas de protección específicas frente a una enfermedad reconocida. Pretende disminuir la probabilidad de ocurrencia de una enfermedad o disminuir su incidencia. La prevención primaria de la enfermedad suele utilizarse como término complementario al concepto de promoción de la salud.

2. Secundaria: su objetivo consiste en lograr un diagnóstico precoz y realizar el tratamiento adecuado con el fin de controlar el progreso de la enfermedad.

3. Terciaria: tiene como propósito mitigar o reducir las consecuencias negativas de las enfermedades y evitar las recaídas. Asimismo, busca mejorar la calidad de vida de las personas.
Por otra parte, Leavell y Clark (1965) distinguieron tres períodos en el curso o historia natural de una enfermedad: 1) el prepatogénico o de susceptibilidad, donde están presentes los factores que favorecen o determinan una enfermedad; 2) el patogénico, donde se observan el estadio presintomático y el de la enfermedad clínica y 3) el de resultados (muerte, incapacidad, estado crónico o recuperación de la salud).

Ahora bien, retomando la trayectoria científica de la autora que nos ocupa, cabe recordar que María Martina Casullo realizó sus primeras investigaciones en el campo de la epidemiología psiquiátrica. Más aún, entre 1964 y 1965 fue psicóloga de la Sección de Epidemiología Psiquiátrica del Instituto Nacional de Salud Mental (INSM). El INSM se había organizado en 1957, en el marco de la renovación psiquiátrica argentina y latinoamericana y fue una de las instituciones responsables de la posterior organización de los servicios de psicopatología en los hospitales generales y de la masiva inclusión del psicoanálisis en las prácticas de salud mental (Vezzetti, 1996; Visacovsky, 2002). Posteriormente, trabajó bajo la supervisión y dirección de Fernando Pagés Larraya, doctor en medicina de la Facultad de Ciencias Médicas de la Universidad de Buenos Aires e Investigador del Consejo Nacional de Investigaciones Científicas y Técnicas, CONICET (Mangiapane \& Gómez Vecchio, 2007; Maristany, 2008). En la década de 1970, Pagés Larraya comenzó a desarrollar el Programa de Investigaciones en Epidemiología Psiquiátrica (PEPSI). El principal aporte de dicho proyecto consistió en establecer un mapa epidemiológico de la patología mental del territorio argentino, al que dividió en 25 isoidias culturales. Las investigaciones realizadas por este autor se inscribían en un campo que aproximaba los enfoques de la psiquiatría transcultural, la epidemiología, la antropología y la etnografía. En ese marco, sus estudios en el terreno epidemiológico se caracterizaron por proporcionar un conocimiento aplicable a los problemas sociosanitarios presentes en nuestro país en aquel momento, desde una perspectiva fuertemente cultural y antropológica (Balzano, Barrial Delmonte, Grasso, \& Sanz, 2008). 
De la epidemiología psiquiátrica a la psicología positiva. Historia de la trayectoria científica de María Martina Casullo

Muy pocos estudios han examinado la historia de la investigación científica, especialmente del Consejo Nacional de Investigaciones Científicas y Técnicas (CONICET), durante los años de la dictadura militar iniciada en 1976. Pero tanto las historias oficiales (CONICET, 2006) como las historias críticas coinciden en el crecimiento institucional desmesurado del CONICET, que se apoyaba en la utilización de los recursos de subsidios para variadas actividades financieras (CONICET, 1989; Hurtado, 2010). En dicho contexto la existencia de un programa de investigación, en lugar de un instituto o centro, resultaba atípica.

En cualquier caso, tanto debido a sus enfoques teóricos como por sus concepciones institucionales, Pagés Larraya consideraba el factor social como un elemento indispensable a la hora de definir la epidemiología psiquiátrica. En contraposición a las definiciones clásicas, afirmaba que dicha disciplina centraba sus esfuerzos en el estudio de la enfermedad mental entendida como un fenómeno social. Al mismo tiempo, se ha planteado que su perspectiva epidemiológica sobre las variaciones en salud mental desbordaba el campo de la psiquiatría propiamente dicho. Por tal motivo, su mirada epidemiológica más bien podía ser definida como epidemiología en salud mental (Ferrero, 2000).

También puede observarse esta concepción en el pensamiento de María Martina Casullo. En efecto, el primer capítulo de su libro Las técnicas psicométricas y el diagnóstico psicopatológico se titulaba, precisamente, "Las tareas de prevención y los estudios epidemiológicos en salud mental" (Casullo, 1992). En su opinión, considerar la influencia de los factores psicosociales en la génesis y el desarrollo de las psicopatologías constituía un factor de vital importancia a la hora de realizar estudios epidemiológicos; a su vez, sostenía que dichas investigaciones son un prerrequisito indispensable para implementar posteriormente acciones preventivas en el área de salud mental:

Para poder formular con precisión estrategias orientadas hacia la promoción y la prevención en Salud Mental, es condición necesaria el desarrollo de proyectos de investigaciones epidemiológicas que tengan como unidades de análisis realidades socioculturales distintas o realidades institucionales interesantes. (Casullo, 1992, p. 14)

En esa dirección, enfatizaba también que al hablar de salud y enfermedad no podían desconocerse las diferencias sociales y culturales, presentes aún en las distintas regiones que integraban un mismo país:

Debemos atrevernos a ser capaces de enfrentar ciertos desafíos epistemológicos: diferenciar entre constructos (variables) con características universales o generales (éticas) de aquéllas que son específicas para un grupo social o subcultural (émicas). (Casullo, 1992, p. 14)

\section{Estudios sobre los factores de riesgo en adolescentes}

Numerosas investigaciones han señalado la pluralidad de problemas, de objetos de estudio (desde la mente y el sujeto hasta el comportamiento, desde el organismo hasta la personalidad y la persona) y de abordajes que caracterizaron el surgimiento de la psicología en el último cuarto del siglo XIX, remarcando así los orígenes policéntricos de la disciplina (Danziger, 2006; Klappenbach, 2006b). Sin embargo, los principales representantes del movimiento de la psicología positiva a nivel internacional afirmaban que, particularmente en los Estados Unidos de Norteamérica después de la Segunda Guerra Mundial, la psicología aplicada a la problemática clínica se había centrado principalmente en el estudio de la patología y la enfermedad mental (Seligman \& Csikszentmihalyi, 2000).

En esa dirección, hace poco más de una década, María Martina Casullo afirmaba que en el campo de la psicología podía observarse un "predominio de la mirada clínica por sobre la salugénica" (Casullo, 2000, p. 344). Como consecuencia de ello, esta disciplina habría adoptado como propio el modelo médico y se habría centrado en el estudio de la psicopatología, es decir, las dimensiones clínicas o patogénicas del comportamiento humano. Asimismo, señalaba que los factores que promovían y conformaban la salud psíquica constituían una temática escasamente investigada. 
No es el momento de señalar que esta perspectiva sobre el origen de la psicología ha sido largamente cuestionada por casi toda la historiografía crítica de los últimos treinta años. Más todavía, ya un texto clásico de la historiografía de la ciencia francesa de posguerra señalaba al menos cinco tradiciones de pensamiento diferentes en el surgimiento de la psicología (Canguilhem, 1958).

Lo que interesa para nuestra investigación es que Casullo destacaba los aportes realizados desde el "enfoque salugénico o positivo" (Casullo, 2000, p. 342) al análisis de las variables que permitían un desarrollo sano. En el marco de su propia trayectoria como investigadora, remarcaba que estudiando los factores de riesgo que hacían vulnerables a los adolescentes favoreciendo en ellos la estructuración de procesos psicopatológicos, pudo observar también la presencia de factores protectores o salugénicos que hacían posible un desarrollo sano:

La experiencia personal en el campo de las investigaciones epidemiológicas desde el año 1984 así como la concreción de proyectos de investigación subsidiados por la Universidad de Buenos Aires sobre depresión, riesgo suicida y malestar psicológico en adolescentes, han permitido estudiar el impacto de los factores de riesgo, entendidos como aquellos procesos y situaciones que favorecen la estructuración de patologías, convirtiendo al sujeto en alguien vulnerable. // En el marco de tales investigaciones, ha sido posible constatar que las mismas situaciones pueden inhibir la aparición de lo patológico promoviendo la génesis de situaciones de resistencia (resilience) sobre la base de procesos salugénicos o protectores, que incorporan tanto aspectos personales/subjetivos como ambientales y culturales diversos. (Casullo, 2000, p. 341)

Debe mencionarse aquí que desde el año 1984 Casullo llevó a cabo el proyecto de investigación "Desarrollo y validación de técnicas de evaluación psicológica para su aplicación en investigaciones epidemiológicas", subsidiado por el CONICET. Asimismo, cobra especial relevancia el último proyecto de investigación que dirigió en la Universidad de Buenos Aires: "Compromiso ético, fortalezas humanas y desafíos contextuales específicos: desarrollo de técnicas de evaluación psicológica de propuestas de la psicología salugénica ó positiva" (2004-2007). Como puede observarse, ambos proyectos se ubican en el cruce de dos líneas de investigación psicológica: la epidemiología y la psicología positiva, por un lado, y la evaluación psicológica, por el otro. Este hecho no resulta en modo alguno casual, ya que desde el año 1987, María Martina Casullo fue la Profesora Titular de la asignatura Teoría y Técnica de Exploración y Diagnóstico Psicológico. Módulo I de la Facultad de Psicología de la Universidad de Buenos Aires.

En ese contexto general y partiendo de una perspectiva epidemiológica-preventiva, las investigaciones realizadas por Casullo desde el año 1985 tuvieron como propósito final el diseño y la adaptación de técnicas de evaluación psicológica (cuestionarios y escalas autoadministrables) que permitían detectar, en el ámbito de las instituciones educativas, aquellos adolescentes que se encontraban en situación de riesgo.

Sus estudios se focalizaron en la atención primaria de la salud, concepto ampliamente difundido a partir de la Conferencia de Alma-Ata, realizada por la Organización Mundial de la Salud en el año 1978. Este concepto aludía a cuatro grandes ejes: a) la extensión de la cobertura buscando que todos los ciudadanos pudieran acceder a los servicios del sistema de salud; b) la necesidad de contar con profesionales (médicos, psicólogos, docentes, enfermeros y líderes comunitarios) capacitados para trabajar en dicho campo; c) la importancia de promover la participación de los propios miembros de la comunidad y d) el logro de la articulación entre los diferentes sectores involucrados (Casullo, 1998).

$\mathrm{Al}$ respecto, Casullo señalaba lo siguiente en su libro Adolescentes en riesgo. Identificación y orientación psicológica, publicado por la Editorial Paidós en el año 1998:

Este trabajo hace referencia a investigaciones desarrolladas en nuestro país desde 1985 como parte de las actividades que la autora realiza en su condición de miembro de la Carrera de Investigador del CONICET (Consejo Nacional de Investigaciones Científicas y Técnicas) así como a las concretadas en la Facultad 


\section{De la ePIDEMIOlogía PSIQUiÁtrica a la pSicología POSITIVA. Historia}

de la trayectoria científica de María Martina Casullo

de Psicología, subsidiadas por la Secretaría de Ciencia y Técnica de la Universidad de Buenos Aires, con un enfoque epidemiológico-preventivo y focalizadas en lo que la Organización Mundial de la Salud denomina “atención primaria”. (Casullo, 1998, p. 15)

En este marco, focalizó sus esfuerzos en el estudio del proceso salud-enfermedad. Asimismo, dada la naturaleza bio-psico-social de los problemas que afectan al ser humano, adoptaba una perspectiva teórica integradora para el análisis los mismos:

Abordar el tema de la adolescencia en riesgo supone necesariamente adoptar una perspectiva teórica integradora, que incorpore las aportaciones del Psicoanálisis, la Psicología Cognitiva, la Psicología Cultural y Social como los que brindan la Sociología y la Neurobiología. // Los adolescentes, objeto de nuestro estudio, son en sí mismos sistemas complejos integrados por diversos subsistemas: biológico, psicológico, cultural, social. (Casullo, 1998, p. 23)

Entre los factores de riesgo en la adolescencia incluía los siguientes comportamientos: uso de tabaco, problemas con la alimentación, consumo de alcohol y de drogas, actitudes e ideaciones suicidas, accidentes no intencionales, delincuencia, violencia, embarazo adolescente, enfermedades de transmisión sexual, abandono del hogar paterno, fracasos en los aprendizajes escolares y episodios depresivos.

Si bien los factores de riesgo en la adolescencia constituían el objeto de sus investigaciones, Casullo destacaba al mismo tiempo la importancia de estudiar los factores protectores o salugénicos en dicha población:

Para las Ciencias de la Salud, entre las que se incluye la Psicología, es necesario conocer la proporción de sujetos que padecen determinada patología en un contexto sociocultural y un tiempo histórico específicos, así como poder estimar la franja de población en riesgo y la que puede considerarse "sana". // En los distintos capítulos hemos destacado que la escuela de nivel medio, oficial o privada, es una unidad de análisis fundamental, tanto para hacer estudios epidemiológicos como para implementar tareas relacionadas con la atención primaria de la enfermedad mental y las diversas formas de su prevención. (Casullo, 1998, p. 106-107)

Por una parte, las técnicas de evaluación psicológica daban cuenta de la presencia de síntomas y signos indicativos la existencia de trastornos psicopatológicos; pero, al mismo tiempo, permitían diferenciar a aquellos sujetos que habían alcanzado un determinado nivel de bienestar psicológico.

En las primeras investigaciones que comenzó a diseñar Casullo, relacionadas con el bienestar psicológico en adolescentes, puede observarse claramente que incorporaba los aportes de la teoría de Carol Ryff:

El bienestar psicológico (la salud mental) puede definirse en términos de seis campos o dimensiones conceptualmente diferentes (Schmutte \& Ryff, 1997):

- Actitud positiva hacia la vida personal presente y pasada (Autoaceptación).

- Capacidad para manejar con eficacia los vínculos psicosociales (Dominio).

- Presencia de relaciones afectivas significativas (Afectos positivos)

- Creencias acerca del sentido de la vida (Proyecto de Vida).

- Sentimientos positivos hacia el crecimiento y la madurez (Desarrollo personal).

- Sentido de autodeterminación (Autonomía).

- (Casullo, 1998, p. 16)

Por otra parte, Casullo rescataba también las contribuciones de Aaron Antonovsky, provenientes del campo de la sociología de la medicina:

El interés por el denominado enfoque salugénico nos llevó a contactarnos con la obra del psicólogo israelí A. Antonovsky, quien ya en el año 1971 estudió a un grupo de mujeres sobrevivientes de campos de concentración, veinticinco años después. En 1979 publica el libro HEALTH, STRESS AND CO. PING donde analiza las variables que ha estudiado: bienestar psicológico, satisfacción con el rol, tipo de relaciones familiares. (Casullo, 2000, p. 341) 
Antonovsky sostenía que el mero hecho de vivir era estresante y centraba su interés en el estudio de aquellas fortalezas que permitían afrontar las situaciones ansiógenas y conflictivas; denominó a dichos factores recursos generalizados para resistir (GRRs). El sentido de coherencia (SOC) fue otro concepto clave dentro de su modelo, definido como una orientación global del individuo que expresaba el grado en el que las personas confiaban en que los estímulos del medio eran explicables y predecibles, que contaban con los recursos para afrontarlos y que valía la pena involucrarse y comprometerse para hacer frente a dichas demandas (Antonovsky, 1988).

Una de las primeras referencias a Antonovsky que puede rastrearse en la obra de Casullo paradójicamente se encuentra vinculada al estudio del riesgo suicida en adolescentes. En dicho marco, analizaba el papel del apoyo social, destacando principalmente dos perspectivas: por una parte, aquellas teorías según las cuales el apoyo actuaba básicamente en las situaciones de estrés, amortiguando las consecuencias negativas de las mismas y, por la otra, aquellos modelos que sostenían que el apoyo social era beneficioso en sí mismo, aumentando las posibilidades del sujeto de sentirse bien. Dentro de estos últimos modelos remarcaba los aportes del autor israelí:

Sin duda el representante más genuino de este enfoque es el psicólogo social israelí Antonovsky $(1979,1987)$, quien destaca que la vida es un proceso en sí mismo estresante, las situaciones vitales con bajo estrés "son más imaginarias que reales". Para este autor el ser un miembro integrado a la comunidad de pertenencia, con buenos lazos sociales, es un buen predictor de la disminución de malestares y sintomatologías. (Casullo, 1998, p. 105)

Por último, debemos hacer referencia a lo que Casullo denominaba el riesgo del talento no identificado. Afirmaba que los adolescentes talentosos y creativos podían llegar a constituir un grupo de riesgo. Dichos sujetos necesitaban un contexto que fuera significado como desafiante y contar con apoyo afectivo y social para poder desarrollar sus capacidades; de lo contrario, podían transformarse en personas aisladas llegando a padecer problemas de conducta o trastornos de personalidad (Casullo, 1998). Al mismo tiempo, ponía de manifiesto que, dado el predominio del modelo médico en nuestra disciplina, escasos estudios se habían focalizado en este grupo.

La Psicología siempre se ha interesado por el estudio del sujeto diferente. Pero, cabe reconocer que se sabe más respecto de las carencias (desviaciones negativas como la deficiencia mental) que del aspecto positivo de ese espectro. Este hecho es parte del fenómeno global al que hicimos referencia al comienzo de este trabajo, del predominio de la mirada clínica por sobre la salugénica. Los talentosos y creativos son simplemente seres privilegiados que deben ser admirados; no tienen características de personalidad y comportamiento que interesen ser comprendidos y explicados. (Casullo, 2000, p. 344)

A partir de lo expuesto anteriormente, entonces, puede observarse cómo dichos estudios epidemiológicos sobre depresión, riesgo suicida y malestar psicológico en adolescentes, centrados en la atención primaria de la enfermedad mental y su prevención (Casullo, 1998), constituyeron el detonante para el análisis de los procesos salugénicos o protectores que impedían que las personas enfermaran aun frente a situaciones ambientales adversas (Casullo, 2000).

Desde una perspectiva que ya se ha discutido para el caso de Casullo, en el año 2001 Norma Contini sostenía que durante la segunda mitad del siglo XX había predominado en la psicología un enfoque psicopatológico centrado en el malestar, los comportamientos desajustados y el déficit; asimismo, señalaba que este hecho podía deberse a la necesidad de dar respuesta a los problemas de salud existentes en los países en desarrollo, entre ellos la Argentina.

Es poco alentador admitir que la consigna de la Conferencia de Alma Ata (OMS, 1978), "Salud para todos en el año 2000", no se ha cumplido plenamente en la Argentina, como tampoco en otros países de los llamados "emergentes". La Conferencia Mundial 
De la ePidemiología pSiQuiátrica a la psicología positiva. Historia DE la trayectoria Científica de María Martina Casullo

de Promoción de la Salud (OPS, 2000) señala que sigue vigente esta noble meta, a la que ahora se suma otro concepto, el de equidad en el acceso a la salud y a la calidad de vida. (Contini, 2006, p. 24)

No obstante ello, afirmaba que desde la década de 1960 podía observarse el cambio hacia un nuevo paradigma basado en el modelo salugénico propuesto por Aaron Antonovsky, al mismo tiempo sostenía que dicho modelo superaba ampliamente al enfoque psicopatológico: "la orientación salugénica focaliza el estudio de los orígenes de la salud; plantea como pregunta clave por qué un sujeto se sitúa en el extremo positivo del continuum salud/ enfermedad" (Contini, 2001, p. 308).

Por otro lado, Contini incorporaba también los aportes del relativismo cultural enfatizando que no podía describirse el comportamiento de un sujeto, evaluándolo etnocéntricamente desde la propia cultura del investigador. Desde esta perspectiva, existían, por una parte, los universales culturales, ubicándose en este grupo los conceptos de salud y calidad de vida (enfoque etic). Por la otra, era importante considerar cómo cada grupo cultural definía dichos conceptos, en otras palabras, hacía referencia a los comportamientos específicos de cada grupo (enfoque emic). En esa dirección, sostenía que debían estudiarse los estándares internos de cada sociedad respecto al bienestar y la satisfacción (Contini, 2001).

En una dirección coincidente, tal como fuera mencionado anteriormente, Casullo remarcaba que para realizar una generalización válida de los resultados, era necesario analizar previamente el grado de universalidad psicológica de los constructos y variables estudiados (Casullo \& Fernández Liporace, 2006). Por tal razón, tanto Casullo como Contini subrayaban la importancia de considerar la influencia del contexto cultural a la hora de encarar programas de intervención centrados en la prevención y la promoción de la salud.

\section{Conclusiones}

En sus investigaciones sobre los factores de riesgo en la adolescencia, realizadas desde una perspectiva epidemiológica-preventiva, María Martina Casullo vinculó los estudios en el campo de la psicología con las problemáticas sociales concretas que presentaban los sujetos de este grupo en la Argentina. Dichos estudios epidemiológicos sobre depresión, riesgo suicida y malestar psicológico en adolescentes, centrados en la atención primaria de la enfermedad mental y su prevención (Casullo, 1998), la llevaron a analizar los procesos salugénicos o protectores que impedían que las personas enfermaran aun frente a situaciones ambientales adversas. En definitiva, estas investigaciones pueden considerarse el principal antecedente del campo que posteriormente Casullo (2000) definiera como psicología salugénica o positiva a finales del siglo XX.

Merece señalarse que las posiciones de Casullo se correspondían con aproximaciones análogas dentro del contexto latinoamericano. En Brasil, por ejemplo, Silvia Koller afirmaba que todas las situaciones de la vida, aún las más adversas, podían constituir tanto factores de riesgo como protectores. En esa dirección destacaba la importancia de las investigaciones sobre la resiliencia, entendida como "la capacidad del individuo de adaptarse positivamente a los contextos de riesgo y adversidad". En ese proceso, jugaban un papel central las capacidades y fortalezas de los sujetos que hacían posible un desarrollo sano. Al mismo tiempo, retomando los aportes de la teoría bioecológica de Urie Bronfenbrenner, remarcaba la influencia del contexto social y cultural en el desarrollo de las personas (Dell'Aglio, Koller, \& Yunes, 2006; Paludo $\&$ Koller, 2007).

Por su parte, en Perú, Reynaldo Alarcón (2009) subrayaba el espacio que habían ganado en los países latinoamericanos las investigaciones sobre la resiliencia. Planteaba que este hecho se debía a que involucraban variables de relevancia social presentes en nuestro contexto, entre ellas, la pobreza crítica. La resiliencia aludía precisamente a la capacidad del individuo de interactuar dinámicamente con los factores de riesgo presentes en las situaciones adversas, superando y saliendo fortalecido de las mismas. En tal sentido, remarcaba la importancia de las intervenciones psicológicas destinadas a pro- 
mover el bienestar subjetivo, vale decir, la felicidad de las personas.

En cualquier caso, puede observarse el temprano interés existente en América Latina por el estudio de las variables salugénicas de la personalidad, investigaciones que pueden enmarcarse dentro de lo que internacionalmente se viene denominando el movimiento de la psicología positiva (Castro Solano, 2012).

Para finalizar, es interesante reslatar algunas de las principales contribuciones que María Martina Casullo realizó en los últimos años de su carrera. Entre ellas, propuso la noción de capital psíquico definiéndolo como el "conjunto de factores y procesos que permiten aprender a protegerse y sobrevivir, a generar fortalezas personales" (Casullo, 2005, p. 61). Dicho constructo está integrado por las siguientes capacidades, habilidades, actitudes y valores:

1. Capacidades cognitivas para adquirir y usar formas de conocimiento: creatividad, curiosidad, motivación para aprender y sabiduría.

2. Capacidades emocionales para desarrollar proyectos superando obstáculos: honestidad, autoestima, persistencia y resiliencia.

3. Habilidades cívicas para lograr una mejor participación ciudadana: liderazgo, lealtad, compromiso y prudencia.

4. Capacidades para establecer vínculos interpersonales: inteligencia emocional, amor, sentido del humor, empatía y altruismo.

5. Sistema de valores como metas que orientan los comportamientos: sentido de justicia, capacidad para perdonar, gratitud y espiritualidad.

Al mismo tiempo, enfatizaba la necesidad de estudiar las capacidades y las fortalezas individuales teniendo en cuenta las características del contexto social y cultural. En este sentido, afirmaba que los temas que constituían el objeto de estudio de la psicología positiva debían ser abordados desde distintos niveles de análisis: subjetivo, psicosocial y sociocultural (Casullo, 2008).

En el prólogo de su último libro Prácticas en psicología positiva, Casullo sintetizaba de la siguiente manera la verdadera misión de esta corriente psicológica:
(...) se trata ahora de ser capaces de integrar una mirada que tome en cuenta el capital psíquico humano con el que cada sujeto cuenta, con la consideración de sus fallas, fracasos, dolores y carencias. Si bien es misión de la Psicología ocuparse del estudio de problemas en el campo de las patologías mentales, es imprescindible que focalice su quehacer científico y profesional en investigar las estrategias que posibilitan el desarrollo de un proyecto de vida sano, las fortalezas y virtudes, los talentos, superando sus ataduras a un viejo modelo médico centrado sólo en el análisis de enfermedades y malestares. (Casullo, 2008, pp. 9-10)

\section{Referencias}

Alarcón, R. (2009). Psicología de la felicidad. Introducción a la psicología positiva. Lima: Editorial Universitaria.

Antonovsky, A. (1988). Unraveling the mystery of health. How people manage and stay well. San Francisco: Jossey-Bass Publishers.

Ball, L. C. (2012). Genius without the "great man": New possibilities for the historian of psychology. History of Psychology, 15(1), 72-83.

Balzano, S. M., Barrial Delmonte, E., Grasso, L., \& Sanz, P. (2008). Obituarios. Dr. Fernando Pagés Larraya (1923 - 2007). Interdisciplinaria, 25(1), 121-123.

Bang, C. (2014). Estrategias comunitarias en promoción de salud mental: construyendo una trama conceptual para el abordaje de problemáticas psicosociales complejas. Psicoperspectivas, 13(2), 109-120.

Canguilhem, G. (1958). Qu'est-ce que la psychologie. Revue de Métaphysique et de Moral, 63(1), 12-25.

Caplan, G. (1973). Principios de psiquiatría preventiva. Buenos Aires: Paidós.

Casullo, M. M. (1992). Las técnicas psicométricas y el diagnóstico psicopatológico. Buenos Aires: Lugar Editorial.

Casullo, M. M. (1996). Enfoques para el abordaje de los contenidos básicos comunes desde la psicología. En L. B. Archideo, M. M. Casullo, A. Fleitas Ortíz de Rozas, E. I. Groisman, O. Guariglia, G. Obiols \& S. B. Decibe (Eds.), Formación ética y ciudadana (pp. 73-91). Buenos Aires: Ministerio de Cultura y Educación de la Nación. 


\section{De la ePIDEMIOlogía PSIQUiÁtrica a la pSicología POSITIVA. Historia}

de la trayectoria científica de María Martina Casullo

Casullo, M. M. (1998). Adolescentes en riesgo. Orientación e identificación. Buenos Aires: Paidós.

Casullo, M. M. (2000). Psicología salugénica o positiva. Algunas reflexiones. Anuario de Investigaciones, 8, 340-346.

Casullo, M. M. (2005). El capital psíquico. Aportes de la psicología positiva. Psicodebate. Psicología, Cultura y Sociedad, 6, 59-71.

Casullo, M. M. (2007). Currículum vitae. Manuscrito no publicado.

Casullo, M. M. (2008). [Prólogo]. En M. M. Casullo (Comp.), Prácticas en psicología positiva (pp. 9-10). Buenos Aires: Lugar Editorial.

Casullo, M. M., \& Fernández Liporace, M. (2006). Las propuestas de la psicología positiva. ¿Universales psicológicos o particulares de una visión cultural? Anuario de Investigaciones, 14, 261-268.

Castro Solano, A. (2010). Concepciones teóricas acerca de la psicología positiva. En A. Castro Solano (Comp.), Fundamentos de psicología positiva (pp. 17-41). Buenos Aires: Paidós.

Castro Solano, A. (2012). La psicología positiva en América Latina. Desarrollos y perspectivas. Psiencia. Revista Latinoamericana de Ciencia Psicológica, 4(2), 108-116.

Christopher, J., Richardson, F., \& Slife, B. (2008). Thinking through positive psychology. Theory $\mathbb{E}$ Psychology, 18(5), 555-561.

Consejo Nacional de Investigaciones Científicas y Técnicas. (1989). Informe sobre investigaciones de hechos ocurridos en el CONICET (Consejo Nacional de Investigaciones Científicas y Técnicas). Período 19761983. Buenos Aires: EUDEBA.

Consejo Nacional de Investigaciones Científicas y Técnicas. (2006). CONICET: ciencia y tecnología para el desarrollo. Buenos Aires: Edición Nacional Editora \& Impresora.

Conti, N. A. (2010). Algunas reflexiones en torno a la corriente de psiquiatría basada en evidencias y su impacto en la psiquiatría contemporánea. VERTEX. Revista Argentina de Psiquiatría, 21(94), 444-449.

Contini, N. (2001). Hacia un cambio de paradigma: de la psicopatología al bienestar psicológico. Acta Psiquiátrica y Psicológica de América Latina, 47(4), 306-315.
Contini, N. (Comp.). (2006). Pensar la adolescencia hoy. De la psicopatología al bienestar psicológico. Buenos Aires: Paidós.

Danziger, K. (1979). The social origins of modern psychology. En A. R. Buss (Ed.), Psychology in social context (pp. 25-44). New York: Irvington Publishers.

Danziger, K. (2006). Universalism and indigenization in the history of modern psychology. En A. Brock (Ed.), Internationalizing the history of psychology (pp. 208-225). New York: New York University Press.

Dell'Aglio, D. D., Koller, S. H., \& Yunes, M. A. M. (2006). Resiliência e psicologia positiva: interfaces do risco à proteção. São Paulo: Casa do Psicólogo.

Diener, E. (2003). What is positive about positive psychology: The Curmudgeon and Pollyana. Psychological Inquiry, 14(2), 115-120.

Ferrero, A. (2000). El concepto de epidemiología psiquiátrica. Un recorrido histórico. Acta Psiquiátrica y Psicológica de América Latina, 46(1), 75-79.

Gancedo, M. (2008). Historia de la psicología positiva. Antecedentes, aportes y proyecciones. En M. M. Casullo (Ed.), Prácticas en psicología positiva (pp. 11-38). Buenos Aires: Lugar Editorial.

Greenberg, J., \& Getting, V. (1957). The medical officer's bookshelf on epidemiology and evaluation. American Journal of Public Health, 47(4), 401-408.

Harris, B. (1999). Repoliticizing the history of psychology. En D. Fox \& I. Prilleltenskt (Eds.), Critical psychology. An introduction (pp. 21-35). London: Sage Publications.

Held, B. S. (2004). The negative side of positive psychology. Journal of Humanistic Psychology, 44(1), 9-46.

Hurtado, D. (2010). La ciencia argentina. Un proyecto inconcluso: 1930-2000. Buenos Aires: Edhasa.

King, L. A. (2003). Some Truth behind the Trombones? Psychological Inquiry, 14(2), 128-131.

Klappenbach, H. (2006a). Periodización de la psicología en Argentina. Revista de Historia de la Psicología, 27(1), 109-164.

Klappenbach, H. (2006b). Construcción de tradiciones historiográficas en psicología y psicoanálisis. Psicologia em Estudo, 11(1), 3-17.

Leavell, H. R., \& Clark, F. (1965). Preventive medicine for the doctor in his community. NewYork: Mc. Graw Hill. 
Leighton, A. H., Clausen, J. A., \& Wilson, R. (1957). Exploration social in psychiatry. London: Tavistock.

Leighton, A. H., \& Hughes, J. K. (1961). Culture as causative of mental disorders. En Milbank Memorial Fund. Causes of mental disorders, a review of epidemiological knowledge (pp. 341-366). New York: Author.

Lilienfeld, A. (1957). Epidemiological methods and influences in studies of non infectious diseases. Public Health Report, 72(1), 51-60.

Linley, A., Joseph, S., Harrington, S., \& Wood, A. M. (2006). Positive psychology: Past, present, and (possible) future. The Journal of Positive Psychology, 1(1), 3-16.

Mangiapane, F., \& Gómez Vecchio, R. (7 de septiembre de 2007). Psicología positiva. Entrevista a María Martina Casullo [Blog. Noticias en Salud Mental. El dolor es inevitable, el sufrimiento es opcional]. Recuperado de http://notisam.wordpress. com/2007/09/07/psicologia-positiva-entrevista-amaria-martina-casullo/

Mariñelarena-Dondena, L. (2012a). Recepción y desarrollo de la psicología positiva en la Universidad de Buenos Aires (1998-2008). Psiencia. Revista Latinoamericana de Ciencia Psicológica, 4(2), 76-83.

Mariñelarena-Dondena, L. (2012b). Surgimiento y desarrollo de la Psicología Positiva. Análisis desde una historiografía crítica. Psicodebate. Psicología, Cultura y Sociedad, 12, 9-22.

Mariñelarena-Dondena, L., \& Gancedo, M. (2011). La psicología positiva: su primera década de desarrollo. Diálogos. Revista Científica de Psicología,
Ciencias Sociales, Humanidades y Ciencias de la Salud, 2(1), 67-77.

Maristany, M. (2008). Homenaje a María Martina Casullo (1940-2008). Revista Argentina de Clínica Psicológica, 17(3), 273-275.

Organización Mundial de la Salud. (1986). Carta de Ottawa para la promoción de la salud. Ottawa: Autor.

Organización Mundial de la Salud. (2004a). Prevention of mental disorders. Efective interventions and policy options (Summary report). Ginebra: Autor.

Organización Mundial de la Salud. (2004b). Promoting mental health. Concepts, emerging evidence, practice (Summary report). Ginebra: Autor.

Paludo, S. S., \& Koller, S. H. (2007). Psicologia positiva: uma nova abordagem para antigas questões. Paidéia, 17(36), 9-20.

Seligman, M. E. P. (2011). Flourish. A visionary new understanding of happiness and well-being. New York: Free Press.

Seligman, M. E. P., \& Csikszentmihalyi, M. (2000). Positive psychology: An introduction. American Psychologist, 55(1), 5-14.

Sternberg, R. J., \& Grigorenko, E. L. (2001). Unified psychology. American Psychologist, 56(12), 1069-1079.

Vázquez, C. (2006). La psicología positiva en perspectiva. Papeles del Psicólogo, 27(1), 1-2.

Vezzetti, H. (1996). Los estudios históricos de la psicología en la Argentina. Cuadernos Argentinos de Historia de la Psicología, 2(1-2), 79-93.

Visacovsky, S. E. (2002). El Lanús. Memoria y política en la construcción de una tradición psiquiátrica y psicoanalitica argentina. Madrid/Buenos Aires: Alianza. 